\title{
Histoires de mégalithes : enquête à Port-Blanc (Saint-Pierre-Quiberon, Morbihan)
}

Megalithic histories: Port-Blanc (Saint-Pierre-Quiberon, Morbihan)

Jean-Noël Guyodo et Audrey Blanchard

\section{(2) OpenEdition}

\section{Journals}

Édition électronique

URL : http://journals.openedition.org/abpo/2772

DOI : $10.4000 /$ abpo. 2772

ISBN : 978-2-7535-3513-8

ISSN : 2108-6443

Éditeur

Presses universitaires de Rennes

Édition imprimée

Date de publication : 30 juin 2014

Pagination : 7-30

ISBN : 978-2-7535-3511-4

ISSN : 0399-0826

\section{Référence électronique}

Jean-Noël Guyodo et Audrey Blanchard, « Histoires de mégalithes : enquête à Port-Blanc (Saint-PierreQuiberon, Morbihan) », Annales de Bretagne et des Pays de l'Ouest [En ligne], 121-2 | 2014, mis en ligne le 30 juin 2016, consulté le 01 mai 2019. URL : http://journals.openedition.org/abpo/2772 ; DOI :

10.4000/abpo. 2772 


\title{
Histoires de mégalithes : enquête à Port-Blanc (Saint-Pierre-Quiberon, Morbihan)
}

\author{
Jean-Noël GuYODO, \\ Audrey BLANCHARD \\ Université de Nantes, Département Histoire de l'art et Archéologie - Laboratoire \\ LARA-Nantes, UMR 6566 CReAAH
}

Est-il encore besoin de rappeler la forte concentration sud-armoricaine de sites funéraires, symboliques et domestiques néolithiques, explorés, fouillés et restaurés très tôt par différents acteurs? peut-être, parce que Félix Gaillard (1832-1910), un des pionniers de la recherche archéologique morbihannaise, est ici l'initiateur de la réflexion autour du complexe mégalithique de Port-Blanc à Saint-Pierre-Quiberon...

Propriétaire de l'Hôtel du Commerce de Plouharnel (Morbihan) dès 1852, son intérêt pour les mégalithes a été en grande partie suscité par la visite régulière d'archéologues, tel l'anglais William Collings Lukis qui a séjourné plusieurs années de suite dans ce qui devient vite le point de ralliement de tous bons initiés qui souhaitaient (re)découvrir les nombreux monuments préhistoriques de la région carnacoise. En découle alors une forte activité de recherche, avec plusieurs dizaines de fouilles et de restaurations de mégalithes à son actif dès la seconde moitié du XIX ${ }^{\mathrm{e}}$ siècle, ainsi que ses collaborations avec les sociétés savantes régionales (société polymathique du Morbihan) et nationales (société d'Anthropologie de Paris, etc.), qui ont permis à ses travaux de prendre de l'ampleur et nous ont permis de recevoir des données textuelles et graphiques de qualité, réunies dans des rapports, souvent édités, relativement complets pour l'époque. Félix Gaillard a certes profité d'un environnement archéologique riche mais aussi d'une émulation réelle entre les différents acteurs œuvrant de la ria d'Étel au golfe du Morbihan, îles comprises. Dans ce secteur, l'activité de terrain ne faiblira plus, depuis les travaux dits anciens (fin $\mathrm{XIX}^{\mathrm{e}}$-début $\mathrm{XX}^{\mathrm{e}}$ siècle) de James Miln, Zacharie Le Rouzic, etc., jusqu'aux plus récents, que ce soit sur le mégalithisme, tant conservé sur la terre ferme que submergé (ex. 
Kerbourgnec à Saint-Pierre-Quiberon) ${ }^{1}$ ou les sites d'habitat (ex. GrohCollé, même commune; figure 1) ${ }^{2}$.

Au nord-ouest de la presqu'île quiberonnaise, le complexe mégalithique de Port-Blanc se situe à proximité de la Pointe du Percho, où des ramassages de surface autour de la maison des douaniers édifiée au XIX ${ }^{\mathrm{e}}$ siècle ont permis de réunir une abondante série lithique témoignant d'une occupation domestique. Le débitage de galets de silex par la technique de la percussion posée sur enclume et l'outillage peu varié (grattoirs, pièces esquillées, etc.) rapprochent ce lot des productions de la fin du Néolithique de l'Ouest de la France ${ }^{3}$.

Propriété de la commune depuis 1992, les architectures funéraires de Port-Blanc ont été classées, par liste, Monument Historique en 1889 sous la référence Mérimée PA00091709. À une quinzaine de mètres $\mathrm{NGF}^{4}$, les deux tombes à couloir se dressent désormais en bordure d'une encoche littorale, au sens de plan de faille incliné entaillé par l'abrasion marine.

Mandaté pour constater la dégradation, de nuit, du site le dimanche 18 février 1883, Félix Gaillard décide, en raison de l'urgence, de débuter la fouille dès le lendemain, lundi 19 février ${ }^{5}$. Cette opération de terrain s'achèvera une semaine plus tard; éclaircissement important puisqu'il permet de souligner la qualité de la fouille et du rendu alors que les durées d'intervention habituelles étaient, à l'époque, souvent beaucoup plus restreintes, même de sa part. Dans son récit original de fouille, Félix Gaillard livre moult informations - souvent négligées - concernant le contexte, l'environnement (notamment dunaire), l'état du monument, les relations stratigraphiques, la provenance des objets (céramiques et lithiques). La découverte exceptionnelle de nombreux ossements humains ainsi que l'évocation détaillée de leurs dépôts, primaires ou secondaires, font aussi l'originalité de cette

1. CAssen, Serge, Vaquero, Jacobo, Les Marches du Palais. Recherches archéologiques sur alignements de stèles et tertres funéraires néolithiques autour de la baie de Quiberon (Morbihan, 2000-2002), Nantes, éd. Taskil-Laboratoire de Préhistoire, Université de Nantes, 2003, 166 p., 135 fig., 4 pl. coul.; CASSEn, Serge, BALtzer, Agnès, Lorin, André, Sellier, Dominique, Boujot, Christine, Menier, David, Rousset, Jean-Marc, " Prospections archéologiques et géophysiques de stèles néolithiques submergées en Baie de Quiberon (Morbihan)", Cahiers d'archéologie subaquatique, n 18, 2010, p. 5-32.

2. GuYodo, Jean-Noël, L'habitat néolithique de Groh-Collé (Saint-Pierre-Quiberon, Morbihan), rapport final d'opération. Rennes, Service régional de l'Archéologie de Bretagne, multigraphié, 2008, 1 vol.

3. GuYODO, Jean-Noël, MARCHAND, Grégor, "La percussion bipolaire sur enclume dans l'Ouest de la France de la fin du Paléolithique au Chalcolithique : une lecture économique et sociale ", Bulletin de la Société préhistorique française, n 102-3, 2005, p. 539-549; BlanCHARD, Audrey, Le Néolithique récent de l'Ouest de la France (IV et ${ }^{\mathrm{e}} \mathrm{II}^{\mathrm{e}}$ millénaires av. J.-C.) : productions et dynamiques culturelles, thèse de doctorat de l'université de Rennes I Beaulieu, 2012, 2 vol., 394 et 249 p.

4. Altitude calculée par rapport au Nivellement Général de la France (NGF).

5. GaIllaRD, Félix, Fouilles des dolmens du Port-blanc à Saint-Pierre-Quibéron, février 1883. Rapport déposé à la Commission des monuments mégalithiques, à la Société d'anthropologie, à Paris, et à la Société polymathique, à Vannes, par Félix Gaillard, Vannes, Impr. Galles, 1883 , in- 8 br., 14 p., 6 pl. 
Histoires de mégalithes : enquête à Port-Blanc

Figure 1 - Localisation des sites mentionnés

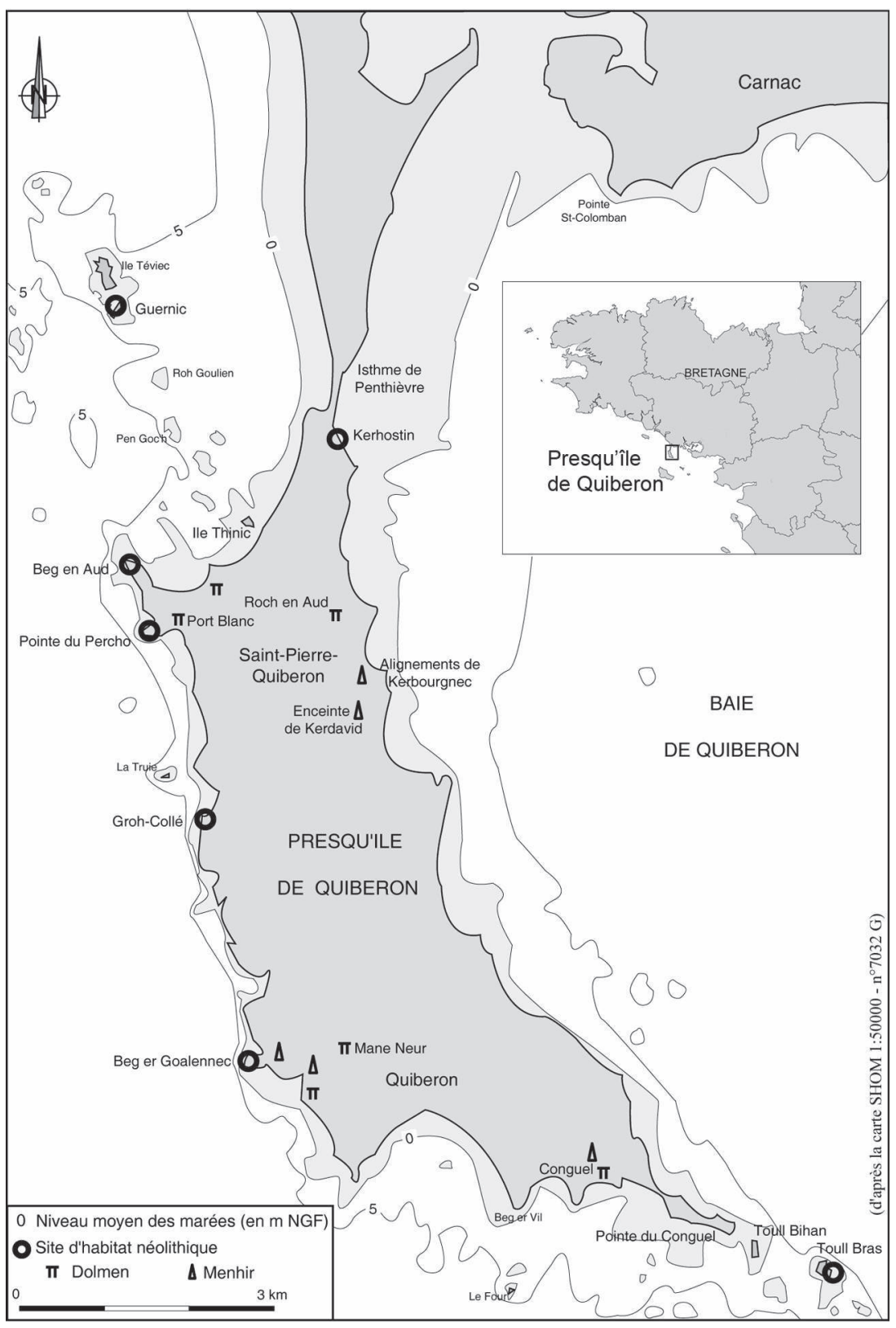

9 
fouille et de ce texte ancien. Les études récentes, réalisées au cours de la dernière décennie, relatives à la culture matérielle ainsi que des données radiométriques, seront également créditées ici. Enfin, les constatations les plus récentes - inédites de surcroît - concernant les thèmes de l'acquisition et la gestion différenciée des matériaux de construction, les techniques employées et une proposition de phasage de ce complexe mégalithique, seront versées au dossier.

\section{Notice nécrologique}

Trois espaces architecturés ont été identifiés en 1883 : deux tombes à couloir (dites " A et B "), de même orientation (ouverture au sud-est), constituées de blocs mégalithiques, et une structure empierrée découverte au pied d'un grand bloc couché identifiée dans la tranchée exploratoire (" annexe C "), ouverte au nord-est, quelques mètres plus loin (figure 2).

Figure 2 - Plan général du site (d'après Gaillard, 1883)

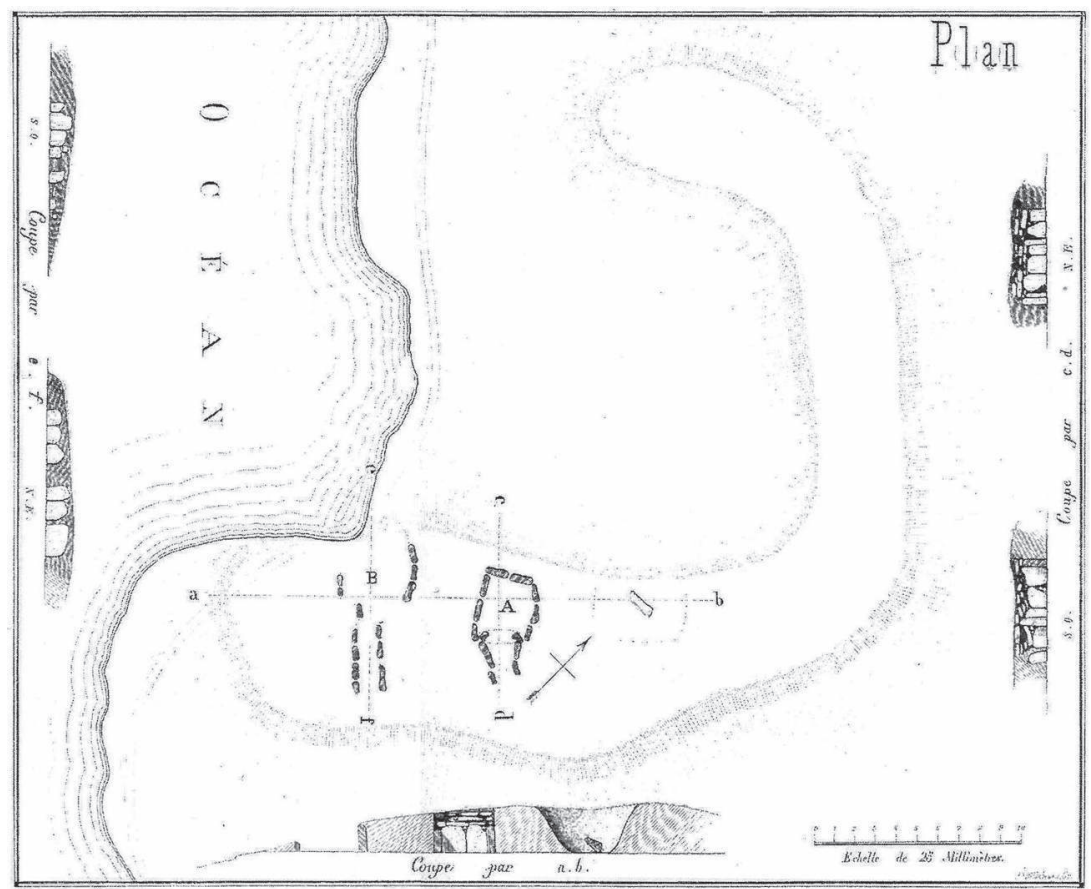

Le premier dolmen fouillé (nord-est, dénommé A) comportait, comme aujourd'hui, treize piliers en place dont huit pour la seule chambre; tous d'une hauteur peu importante (1,20 à 1,30 m). Large de 1,10 m au niveau de 
l'entrée, le couloir de $2 \mathrm{~m}$ de longueur s'ouvre vers le sud-est et débouche, côté opposé, sur une chambre unique de forme presque quadrangulaire ( $2,65 \times 2,55 \mathrm{~m})$. Une seule table de couverture $(2 \times 0,90 \mathrm{~m})$ a été reconnue à l'interface couloir/chambre; elle avait malheureusement été retirée par les pilleurs. La chambre était vraisemblablement couverte par une voûte en encorbellement, alors partiellement conservée. Enfin, et de manière excessive, tous les blocs de l'architecture sont dits roulés par la mer. Le deuxième dolmen (sud-ouest, dénommé B) est distant de $5 \mathrm{~m}$ du précédent, et parallèle au premier. Le couloir, de largeur sensiblement équivalente $(1 \mathrm{~m})$, est tout de même plus long $(3,25 \mathrm{~m})$ et comportait huit piliers, contre quatre désormais. La chambre, peut-être circulaire, de $4 \mathrm{~m}$ de diamètre, était fort endommagée avant la fouille de Félix Gaillard car déjà proche du "précipice ". Aucune mention n'est faite des trois piliers dressés aujourd'hui, côté nord.

Désigné sous le terme poétique de l'époque galgal ${ }^{6}$, le cairn englobant les deux tombes à couloir était préservé sur une hauteur de 1 à 1,50 m et vu comme une entité unique; observation sur laquelle nous reviendrons plus largement. En façade, il est flanqué d'un muret de pierre sèche servant de parement externe au monument, dont il ne reste aujourd'hui que l'assise basale. Les dolmens ayant été peu dégradés avant son intervention, Félix Gaillard constate que les entrées des deux couloirs étaient encore fermées par des blocs de pierres accumulées, suggérant ici leur condamnation, sans doute au terme de leur utilisation. Concernant l'intérieur, " dans ces deux dolmens, et dans les chambres seulement, [...] il existait une couche de petits galets ou cailloux de rivage sur lesquels était posé le dallage de pierres plates ${ }^{7}$ ". Cette couche de petits galets de couleurs variées surmontée d'un dallage, qui ne concerne que les chambres et en aucun cas les couloirs, trouve déjà sous sa plume un premier point de comparaison lorsqu'il rappelle que Gustave de Closmadeuc avait constaté en tout point la même chose en 1866 dans la tombe à couloir de Keriaval à Carnac (Morbihan) ${ }^{8}$, tout comme James Miln lorsque ce dernier fouilla et restaura plusieurs monuments dans la région.

À 5,20 m au nord-est de la tombe à couloir A, un long bloc $(1,70 \times 0,60 \mathrm{~m})$ couché a été découvert lors de l'ouverture d'une tranchée exploratoire complémentaire (dite annexe C). À son pied, un lit de pierres formant une plateforme grossière s'étend sur $0,95 \times 0,95 \mathrm{~m}$, pour $0,40 \mathrm{~m}$ d'épaisseur, sur laquelle ont été trouvés plusieurs crânes et ossements humains.

Si les architectures évoquées, en bon état de conservation, livrent déjà des informations précieuses qui seront développées infra, les relations stra-

6. Galgal : terme à consonance " celtique " alors utilisé pour évoquer une importante masse de pierre, dans le cas présent pour le cairn de l'architecture mégalithique.

7. Gaillard, Félix, Fouilles des dolmens du Port-blanc à Saint-Pierre-Quibéron, février $1883 \ldots$ op. cit., cf. p. 5

8. Closmadeuc, Gustave DE, " Rapport sur les fouilles faites dans les communes de Carnac et de Plouharnel ", Bulletin de la Société polymathique du Morbihan, 1866, p. 91-150. 
tigraphiques relevées tant à l'intérieur qu'à l'extérieur des architectures funéraires offrent des données somme toute peu fréquentes pour l'Ouest de la France. La bonne conservation des ossements humains est la conséquence d'un recouvrement massif du monument par du sable coquillier. Ce niveau dunaire de plus d'un mètre de hauteur est mentionné sur les illustrations anciennes mais a totalement disparu aujourd'hui, ce en un peu plus d'un siècle (figure 3), offrant une vision actuelle très épurée de l'environnement du complexe mégalithique; d'où la volonté pour le Conservatoire du littoral et des rivages lacustres de préserver, aussi, ce secteur.

\section{Figure 3 - Vue du couloir depuis la chambre de la tombe à couloir A (dit dolmen nord-est) de Port-Blanc (gauche, d'après Gaillard, 1883; droite, cliché J.-N. Guyodo 2008)}

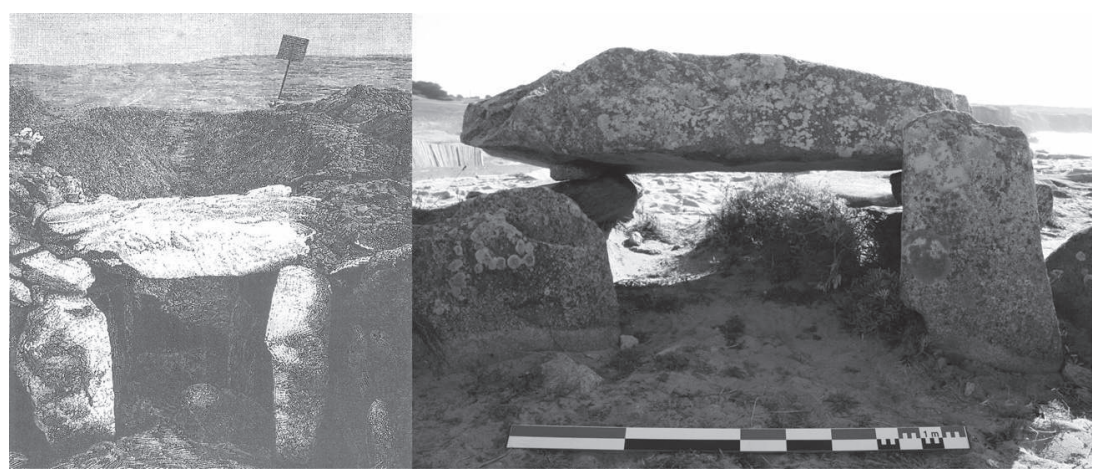

Dans la chambre de la tombe à couloir A, ce sont en définitive deux couches d'ossements humains, séparées par un niveau de pierres plates, qui ont été découvertes. La couche supérieure, de fait la plus récente, réunit notamment deux individus, crânes au nord-est et torses parallèlement allongés au sud-est. Ce qui les distingue au niveau de la position est que le premier a la tête sur le côté gauche et le second sur le côté droit. Le mobilier funéraire est peu important, avec une épingle en os sur le corps de l'un et un poinçon dit en bronze sur ses côtes de l'autre. Alors que la fouille de ce niveau supérieur s'est achevée le 22 février, Félix Gaillard mentionne la découverte, le 26 , de quatre crânes, dont trois se touchant presque, de fémurs amoncelés et de nombreux débris osseux recouvrant le crâne placé dans l'angle, ainsi qu'un cinquième crâne; le tout dans l'angle sud-ouest de la chambre. S'il semble bien s'agir d'un cas classique de réduction de corps, évacués et accumulés le long de la paroi, on peut toutefois se demander quelle est la valeur stratigraphique de ce lot alors que le décalage calendaire mentionné peut ici prêter à confusion et laisser planer un doute sur la relation assurée entre les deux inhumés du milieu de la chambre et la réduction des corps le long de la paroi. Dans la soirée du 22 février, le dallage de 
pierres plates, base de la couche supérieure, est retiré, laissant apparaître une seconde couche (dite inférieure) contenant des restes humains : onze nouveaux crânes et de nombreux ossements mêlés, principalement le long des parois et au fond de la chambre, le tout dispersé sur un nouveau dallage, dit inférieur. Aucune trace de charbon ni de cendre mais, fait rare dans l'Ouest de la France, un des crânes est trépané; la rondelle osseuse retirée mesurant $0,06 \times 0,05 \mathrm{~m}$.

De nombreux restes humains furent également découverts dans la deuxième tombe à couloir (B), plus dégradée et dont l'architecture était en plus mauvais état. Dans le couloir, près de la chambre, c'est un groupe de cinq squelettes qui a été mis au jour (figure 4). Les premiers constats signalent quelques déconnexions anatomiques (corps/crâne) et, plus fréquemment, des corps qui auraient pu avoir été adossés contre les piliers, certains s'étant comme effondrés avec le temps, ou allongés près de la paroi. Dans la chambre, cinq crânes et quelques ossements, accompagnés des débris d'un grand vase et de quelques charbons et traces cendreuses, témoignent une nouvelle fois d'un cas de réduction. Malgré l'évacuation des ossements en partie le long des parois, aucun dépôt n'a été identifié au centre de la chambre. Le niveau fouillé, vraisemblablement unique, recouvrait le dallage basal, qui ne concerne que ce seul espace dans l'architecture.

Figure 4 -
Squelettes
découverts
dans le couloir
de la tombe à
couloir B (dit
dolmen sud-
ouest) de Port-
Blanc (d'après
Gaillard, 1883)

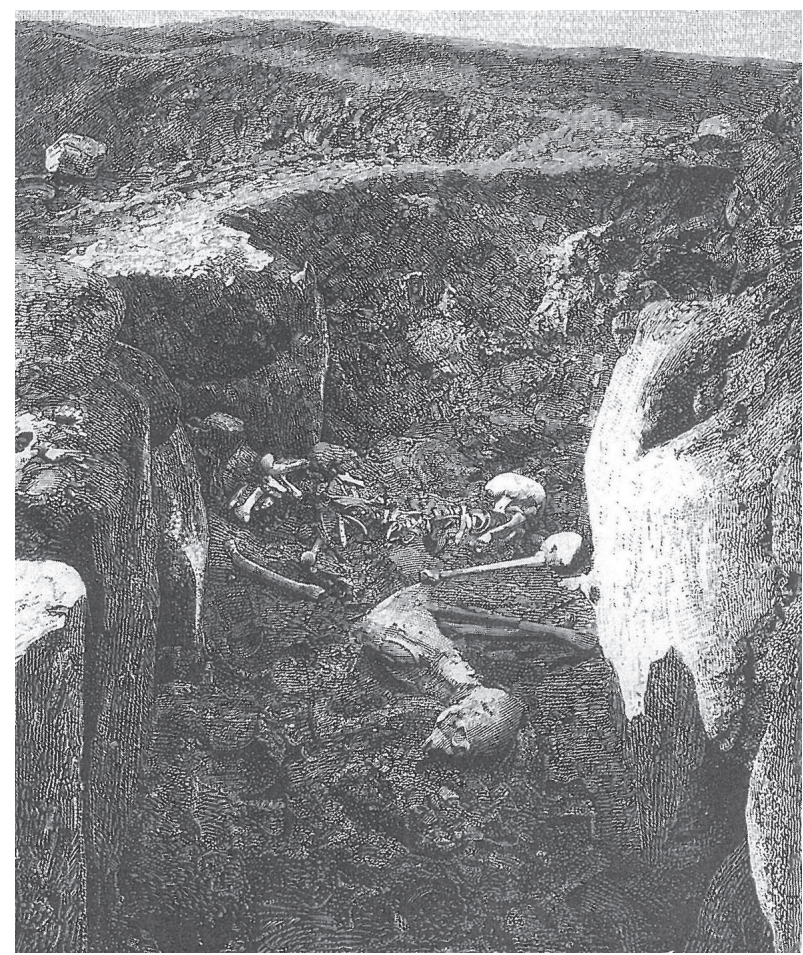


Le dernier lot d'ossements humains a été découvert sur la plateforme de pierres bordant le bloc couché (dit annexe C) découvert dans la tranchée exploratoire, à plus de $5 \mathrm{~m}$ de la plus proche tombe à couloir (A). Les dix crânes agglomérés au-dessus d'un amas d'ossements restreint permettent d'émettre l'hypothèse d'un vraisemblable dépôt externalisé après vidange; du seul dolmen A d'après l'auteur. Si aucun corps entier n'a été reconnu, quelques débris de poteries et percuteurs ont toutefois été ramassés.

Si l'on base le calcul, certes simplifié, sur les seuls ossements les plus cités, c'est-à-dire les crânes, il y aurait au total 37 individus, ce qui ferait déjà de ce lot de loin le plus important ensemble exhumé d'un monument mégalithique sur le Massif armoricain. Et il semblerait que ce chiffre soit a minima puisque Félix Gaillard estime que potentiellement il y avait une quarantaine voire une cinquantaine d'individus rien que dans le dolmen A, où « il a été impossible de se rendre compte exactement du nombre de cadavres qu'il contenait; mais de ce qu'il a été observé sur ce que nous avons fouillé, c'est-à-dire la moitié de la chambre jusqu'au fond $[\ldots]^{9}$ ". Le plus troublant à ce moment du récit, est l'évocation de la seule fouille partielle de la chambre. Si l'exploration de l'autre moitié n'a pas été réalisée, on peut se demander pourquoi, sur la base de ce texte explicite, il n'y a pas eu de reprise des fouilles par la suite et surtout pour quelle raison rien n'est désormais en place dans le monument! Un pillage aurait été dénoncé dans la littérature, or ce n'est pas le cas...

\section{Éléments d'architectures}

De récents travaux d'aménagements, en vue d'une valorisation du site mégalithique et de ses abords, ont été l'occasion d'effectuer, en 2008, quelques observations complémentaires. Depuis 1883, les seuls plans des tombes à couloir ainsi que de leurs élévations ont été dressés à la fin des années 1990 par Thierry Templier (figure 5). L'intervention récente permet d'offrir ici un relevé plus complet de l'architecture (figure 6).

En façade du monument, entre les entrées des tombes à couloir A et B, apparaît un petit coffre quadrangulaire mesurant un mètre de côté, matérialisé sur trois côtés par des dalles verticales, alors que le dernier est le parement basal du cairn, sur lequel il est appuyé. De plus petits blocs entourent ce coffre et participent à la construction. Cette structure n'est pas évoquée par Félix Gaillard, on peut donc se demander si elle a été négligée lors de l'intervention ou si elle est plus récente, en lien avec les travaux légers de restauration de 1884, avant le classement, par liste, Monument Historique en 1889. Les dalles dressées sont en granite, même matériau que l'ensemble architectural, et ne portent aucune trace de débitage (saignée, mortaise), de fragmentation ou de rectification moderne avec des outils métalliques. La forme et la surface correspondent aux normes classiques des coffres

9. Galllard, Félix, Fouilles des dolmens du Port-blanc à Saint-Pierre-Quibéron, février $1883 \ldots$, op. cit., cf. p. 7 
Figure 5 - Plans et élévations des tombes à couloir (levé inédit de T. Templier, archives du laboratoire LARA-Nantes, repris)

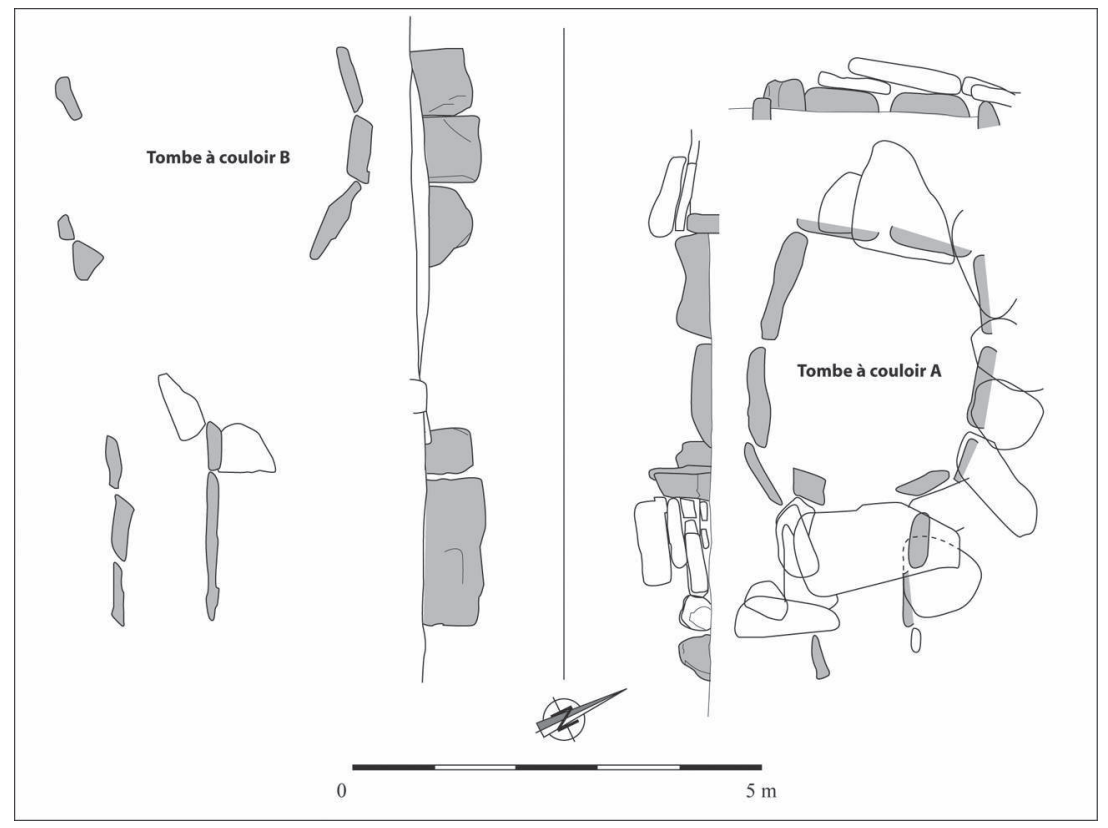

néolithiques, campaniformes ou du début de l'âge du Bronze. Enfin, sa position en connexion avec l'ensemble mégalithique rappelle certains petits coffres ajoutés plus tardivement contre d'autres monuments funéraires (Beg er Goalennec, Quiberon, pour n'en citer qu'un) ${ }^{10}$, et dans certains cas en lien avec des alignements de blocs dressés comme, pour ne citer qu'un exemple, ceux des landes du Moulin de Cojou à Saint-Just (Ille-etVilaine) ${ }^{11}$. Pour toutes ces raisons, ce coffre est vraisemblablement ancien et en place, bien qu'aucun écrit ne relate son exploration. Aucun élément de datation plus précis ne peut être porté au lecteur, si ce n'est qu'il est de fait postérieur au cairn de l'ensemble mégalithique. L'évidente fréquentation ponctuelle campaniforme (début II millénaire avant J.-C.), suggérée par la découverte d'un vase campaniforme au sommet de la couche supérieure de la chambre du monument A, pourrait permettre d'imaginer - si on ne voulait être plus prudent - qui en sont les responsables...

10. GAILLARD, Félix, in Congrès international d'Anthropologie et d'Archéologie préhistoriques, $10^{\mathrm{e}}$ session, Paris 1889, édité en 1891, p. 226-237; RIQUET, Raymond, GuILAINE, Jean, CofFYN, André, "Les campaniformes français. (État actuel des recherches et perspectives)", Gallia préhistoire, t. 6, 1963, p. 63-128.

11. LE Roux, Charles-Tanguy, LECERF, Yannick, GAUTIER, Maurice, « Les mégalithes de SaintJust (Ille-et-Vilaine) et la fouille des alignements du Moulin de Cojou ", Revue archéologique de l'Ouest, nº 6, 1989, p. 5-29. 

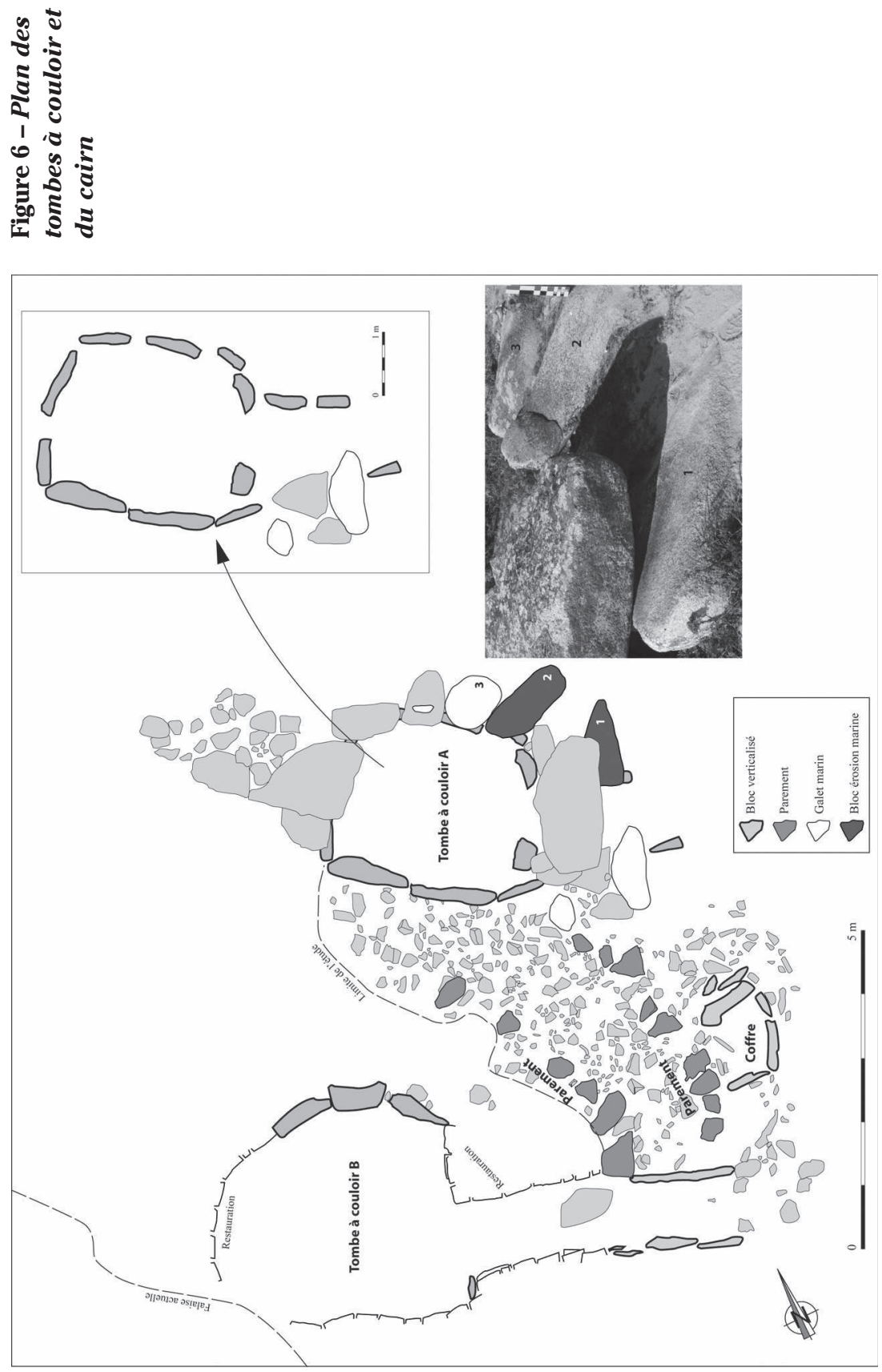
Le cairn, visible juste avant l'aménagement récent, est désormais recouvert d'un niveau de sable qui le camoufle, heureusement sans l'endommager. Les observations ont été faites entre les deux tombes à couloir, seul espace accessible lors notre intervention de 2008. Les assises basales de deux parements s'appuyant, côté sud, sur les piliers du couloir du monument B, sont ici conservées sur une longueur de $5 \mathrm{~m}$. Elles s'interrompent clairement à l'approche du monument A. Cette nette rupture du cairn primaire de la tombe $\mathrm{B}$ correspond à vraisemblablement à un démontage partiel, rendu nécessaire pour bâtir la seconde tombe à couloir (A) qui n'intègre que partiellement le cairn existant et est de fait plus récente. Bien que de formes et d'orientations similaires, les deux tombes à couloir ne sont donc pas strictement contemporaines, ni englobées dans un seul et même cairn, comme cela a été malheureusement souvent dit depuis les premiers écrits de Félix Gaillard ${ }^{12}$. Il aurait certes été plus simple d'adjoindre la seconde tombe à couloir par accrétion et d'apposer son enveloppe externe sur la première, mais il semble que dans le cas présent le surcroît de travail n'ait pas été un frein à l'investissement. Cette observation permet désormais de rendre plus justement compte du phasage chronologique de l'ensemble.

Les cas de transformation d'architectures mégalithiques ne sont pas rares dans l'Ouest de la France. Concernant l'ensemble architectural, plusieurs exemples d'extension par accrétion et adjonction de chambres existent. C'est notamment le cas pour la nécropole mégalithique du Souc'h (Plouhinec, Finistère) ${ }^{13}$, correspondant au final à l'adjonction successive de tombes à couloirs, ainsi qu'à Barnenez (Plouezoc'h, Finistère) ${ }^{14}$, avec l'agrégation de deux grandes architectures recouvrant chacune plusieurs tombes à couloir. Dans le même ordre d'idée, des modifications internes de monuments déjà construits, par insertion de pierres dressées - parfois isolément - dans ou le long du couloir (L'île Longue, Morbihan) ${ }^{15}$ ou la chambre (Barnenez, chambre $\mathrm{H})^{16}$ sont évoquées dans la littérature, ainsi que des témoignages d'accidents comme la chute de dalles et de piliers (chambre II du Petit-Mont à Arzon, Morbihan) ${ }^{17}$. Il faut aussi souligner les transformations de la forme d'un monument, comme le passage d'un plan circulaire à un plan quadrangulaire pour la chambre 2 du monument $\mathrm{E}$ de

12. Gaillard, Félix, Fouilles des dolmens du Port-blanc à Saint-Pierre-Quibéron, février 1883... op. cit.; L'HELGOUAC'H, Jean, Les sépultures mégalithiques en Armorique, thèse de doctorat d'État, université de Bretagne, Rennes, 1965, 1 vol., 332 p. cf. p. 125.

13. LE Goffic, Michel, « La nécropole mégalithique du Souc'h en Plouhinec ", fascicule de la Journée civilisations atlantiques et archéosciences, Rennes, 2006, p. 24-25.

14. Giot, Pierre-Roland, Barnenez, Carn, Guennoc, Travaux du Laboratoire "Anthropologie, Préhistoire, Protohistoire et Quaternaire Armoricains ", Rennes, 1987, 2 vol.

15. L'Helgouac'H, Jean, Les sépultures mégalithiques en Armorique..., op. cit.

16. LAPORTE, Luc, JALLOT, Luc, SOHN Maïténa, « Mégalithismes en France. Nouveaux acquis et nouvelles perspectives de recherche ", Gallia Préhistoire, 53, 2011, p. 289-338, cf. p. 309.

17. LeCoRnec, Joël, Le Petit Mont, Arzon, Morbihan, Documents archéologiques de l'Ouest, Rennes, 1994, 110 p. 
la nécropole mégalithique de Bougon (Deux-Sèvres) et plus près de l'actuel propos les modifications apportées aux façades des complexes mégalithiques, à l'image de la destruction partielle de la façade du monument B de la nécropole de Champ-Châlon (Charente-Maritime) ${ }^{18}$.

L'étude des matériaux employés est aussi un champ d'investigation, récemment exploré et qui participe désormais de la réflexion ${ }^{19}$, puisque leur diversité, tant en terme d'origine et de provenance que de positionnement, est source d'enseignements. Sans vouloir sombrer (voire s'enliser) trop vite, par facilité, dans le débat sur la nature (couleur, etc.) et la qualité architectonique des matériaux, la première observation concerne ici la " simple " provenance des blocs, d'une part issus d'affleurements et/ou de carrières du plateau et d'autre part prélevés (galets marins) et/ou arrachés (piédestaux) sur l'estran. Cette variation est d'ordre chronologique puisque ces derniers ne sont utilisés que pour la construction de l'architecture funéraire que l'on comprend désormais comme la plus récente (A). Sur le plateau, à proximité des monuments, il y avait toutefois encore la possibilité - comme aujourd'hui - d'extraire des blocs de dimensions similaires soit à partir d'affleurements rocheux distants de quelques centaines de mètres, soit en ouvrant des carrières pour l'occasion, comme cela a été identifié sur plusieurs sites d'habitats, pour certains proches (Groh-Collé à Saint-Pierre-Quiberon, Pen Men à Groix), eux aussi datés du Néolithique récent. Prélever sur l'estran, sans doute en contrebas du site c'est-à-dire à même distance que des affleurements, des blocs à surfaces d'érosion marine manifestes révèle ici un changement de stratégie plus que la rupture de possibilités d'approvisionnement, soit véritablement un choix intentionnel, peut-être culturel plus que technique. La seule réelle différence entre ces faits concerne les problèmes de manutention puisque pour le déplacement de ces galets et piédestaux du lieu de prélèvement jusqu'au site mégalithique la difficulté est accrue. Néanmoins, ces blocs n'excèdent pas quelques dizaines à centaines de kilogrammes : il n'y donc pas véritablement exploit physique pour les remonter en haut de la falaise, surtout en profitant, sur plusieurs centaines de mètres, d'une pente régulière...

Le plan général de cet ensemble mégalithique à deux tombes à couloir parallèles, ouvertes au sud-est et incluses dans un même cairn, n'est pas original puisque l'on recense plus d'une vingtaine de cas similaires dans le Morbihan et en Loire-Atlantique, dont les plus proches exemples morbihannais sont ceux de Er Rohec (La Trinité-sur-Mer), de Kergo-er Rohellec, Kergrim, Mané Gardreine, Kerlagad et Roh-en-Tallec (Carnac) et de Er Pointe

18. Joussaume, Roger, Les tumulus de Champ-Châlon à Benon (Charente-Maritime), Groupe vendéen d'études préhistoriques, t. 42, 2006, 90 p.

19. MENS, Emmanuel, L'affleurement partagé, gestion du matériau mégalithique et chronologie de ses représentations gravées dans le Néolithique moyen armoricain, thèse de doctorat en archéologie de l'université de Nantes, 2002, 3 vol., 795 p.; GuYodo, Jean-Noël, MEns, Emmanuel, dir., Les Premières architectures en pierre en Europe occidentale du ve au II millénaire av. J.-C., Actes du colloque international de Nantes, Musée Thomas Dobrée, 2-4 octobre 2008, Rennes, 2013, PUR, coll. " Archéologie et culture ", 307 p. 
(Saint-Philibert). La complexité de l'ensemble tient en revanche au fait que l'on est face à trois espaces sépulcraux distincts, dont un (annexe C) externalisé. Cette dernière structure connexe correspond à une probable zone de stockage des ossements longs et crânes sur une aire aménagée (plateforme dallée), près d'un bloc couché, utilisée, certes sur la seule base des observations transmises par Félix Gaillard, après vidange de la tombe à couloir A. Le plan relativement similaire des tombes à couloir A et B fait état d'une transformation de l'architecture dans un laps de temps sans doute assez court (début du Néolithique récent), ce qui offre - fait peu fréquent - une chronologie relative en ce qui concerne la construction et du moins l'utilisation des espaces sépulcraux internalisés/externalisés, connus dans d'autres contextes néolithiques (vidanges, réductions de corps, réaménagements intérieurs, etc. ${ }^{20}$, mais rarement dans l'Ouest de la France, en raison du piètre état général des ossements humains.

La fermeture de l'entrée, par des blocs de pierres accumulées à PortBlanc, est un geste déjà observé par Armand du Chatellier qui constate, en 1861, l'obturation de l'entrée, vue comme murée, du dolmen du grand tumulus de Rosmeur (Finistère) ${ }^{21}$. Depuis, d'autres constatations similaires ont été réalisées, que ce soit pour le dolmen central de l'île Carn (Finistère) où les deux extrémités du couloir étaient murées ${ }^{22}$ ou lors de la récente fouille de tombe à couloir transeptée de Château Bu à Saint-Just (Ille-etVilaine) dont l'entrée était condamnée par des dalles de schiste verticalisées $^{23}$. Cette condamnation par obturation de l'accès n'est pas généralisée mais reste une pratique somme toute fréquente à l'échelle régionale, ainsi que dans le sud de la France et la péninsule ibérique.

Concernant la position des couloirs, Jean L'Helgouac'h ${ }^{24}$ signale que les sépultures à chambre simple, symétrique par rapport au couloir, sont peu nombreuses et principalement cantonnées au Morbihan (Petit-Mont à Arzon, Mané Carnaplaye à Saint-Philibert, etc.) à de rares exceptions près (Kerleven à Fouesnant, Finistère ${ }^{25}$ ). D'après le mobilier recueilli, ces monuments ne sont néanmoins pas tous contemporains, certains datant

20. CHAMBOn, Philippe, LECLERC, Jean, dir., Les pratiques funéraires néolithiques avant 3500 av. J.-C., en France et dans les régions limitrophes, Actes de la table ronde SPF de SaintGermain-en-Laye, juin 2001, Société préhistorique française, mémoire 33, 2003, 330 p.; Chambon, Philippe, Les morts dans les sépultures collectives néolithiques en France : du cadavre aux restes ultimes, Paris, $\mathrm{Xxv}^{\mathrm{e}}$ supplément à Gallia Préhistoire, 2003, 395 p.

21. Du Chatellier, Paul, "Les deux tumulus de Rosmeur, pointe de Penmarc'h (Finistère)", Matériaux pour l'histoire primitive de l'homme, t. 10, 1879, p. 145-154.

22. Giot, Pierre-Roland, L'HELgouAc'H, Jean, "Le cairn de l'île de Carn en Ploudalmézeau ", Bulletin de la Société archéologique du Finistère, t. 81, 1955, p. 53-62.

23. BRIARD, Jacques, GAUTIER, Maurice, LEROUX, Gilles, Les mégalithes et les tumulus de SaintJust, Ille-et-Vilaine : évolution et acculturations d'un ensemble funéraire, 5000 à 1500 ans avant notre ère, Paris, éd. du Comité des travaux historiques et scientifiques, 1995, 174 p.

24. L'HelgouAc'H, Jean, Les sépultures mégalithiques en Armorique..., op. cit., cf. p. 50.

25. L'HelgouAC'H, Jean, LE RouX, Charles-Tanguy, « Le cairn mégalithique avec sépultures à chambres compartimentées de Kerleven, commune de Fouesnant (Finistère) ", Annales de Bretagne, vol. 74, 1967, p. 7-52. 
du Néolithique moyen II (fin V ve-début IV ${ }^{\mathrm{e}}$ millénaire av. J.-C.), d'autres plus récents. Enfin, à bien y regarder, le couloir de la tombe A de Port-Blanc n'est pas tout a fait symétrique.

Le portique d'entrée de la chambre de la tombe à couloir A, avec ses piliers décalés, est proche du monument d'Er Run de Keredo (Erdeven, Morbihan) dont le plan a été dressé par William Collings Lukis ${ }^{26}$; ce rapprochement est évoqué par Jean L'Helgouac' $h^{27}$. Ce système de passage rétréci n'est encore une fois pas spécifique à la région, loin de là (sud de la France, péninsule ibérique). Bien que l'on soit, dans les deux cas, face à une chambre de forme à peu près carrée, il faut noter une différence : à Port-Blanc, ces deux piliers ne sont pas couverts par une dalle de couverture, d'où l'hypothèse émise d'un possible recouvrement partiel, du moins à cet endroit, par une voûte en encorbellement ${ }^{28}$ dont aucune assise ne serait-ce que basale n'est identifiable, ni mentionnée dans les écrits de Félix Gaillard. Qu'importe le système de couverture, il faut surtout noter que ces piliers sont en place et intégrés dès l'origine à l'architecture et qu'il ne peut s'agir ici de reprise en sous-œuvre comme cela a été récemment remarqué dans quelques (rares) monuments funéraires contemporains de la fin du Néolithique ${ }^{29}$.

À l'intérieur des deux tombes à couloir A et B, Félix Gaillard a noté la présence de dallages, plus que de pavages, couvrant la totalité des chambres; simple remarque qui pourrait passer pour banale puisque de tels aménagements de surface ont déjà été remarqués dans d'autres monuments régionaux, dès le $\mathrm{XIX}^{\mathrm{e}}$ siècle. Néanmoins, le fait que l'on ait ici deux dallages superposés relève d'une plus grande originalité, tout comme les lits de galets annoncés, dont les rares référents concernent les observations réalisées quelques années plus tôt lors de la fouille des dolmens A et $\mathrm{B}$ de Mané Kerioned à Carnac ${ }^{30}$. Enfin, à Port-Blanc, aucun dallage n'a été identifié dans les couloirs, mais ce n'est pas si étonnant puisque l'aménagement et le soin apporté à ces espaces sont très rares.

26. LUKIS, William Collings, " On some peculiarities in the construction of chambered barrows ", Journal of the British Archaeological Association, 1866, p. 349.

27. L'Helgouac'H, Jean, Les sépultures mégalithiques en Armorique..., op. cit., cf. p. 68.

28. Ibidem. p. 69.

29. GAUTIER, Nicolas, Les monuments à chambre compartimentée et transeptée : étude du cloisonnement des espaces funéraires dans l'Ouest de la France à partir de l'exemple des fouilles effectuées par P.-R. Giot et A.M. Apsimon à Quélarn (Plobannalec, Finistère), mémoire de master 1 Archéologie et Histoire, Univ. Rennes 2 Villejean, 2008, 1 vol., 87 p.; HuEt, Marion, Rôle des orthostates dans les dolmens à couloir de l'Ouest de la France, mémoire de master 1 Archéologie et Histoire, Univ. Rennes 2, 2008, 1 vol., 98 p.; LAPORTE, Luc, "Restauration, reconstruction, appropriation; évolution des architectures mégalithiques dans l'Ouest de la France, entre passé et présent ", Munibe, t. 32, 2010, p. 120-150.

30. Galles, René, Gressy, Docteur M., De Closmadeuc, Gustave, "Rapport sur les fouilles faites par la Société Polymathique dans les communes de Carnac et de Plouharnel ", Bulletin de la Société polymathique du Morbihan, 1866 (1867), p. 91-101. 


\section{Chronologies}

Le premier point d'accroche est lié aux précieuses informations offertes par Félix Gaillard concernant la localisation du complexe mégalithique, édifié sur un sol sableux - correspondant à la dune ancienne - et partiellement recouvert par un sable garni de coquilles (dune récente). Ce détail n'est pas anodin car il revient souvent sous sa plume : pour lui les vestiges anciens, néolithiques, de la presqu'île quiberonnaise se trouvent sous cette dune récente, ce qui explique la recherche ciblée, souvent fructueuse, qui lui permit de découvrir de nombreux sites. Au moment de la construction des premières typologies régionales des architectures en pierres, notamment les formes ubiquistes comme les coffres de pierres - pouvant tout autant dater du Néolithique, de l'âge du Bronze que de l'âge du Fer - ce moyen d'évaluer l'ancienneté des gisements lui offre la possibilité de rentrer dans le débat scientifique de cette fin du XIX ${ }^{e}$ siècle, lorsque les écrits polémiques s'échangent parfois entre chercheurs pour la datation de certains sites ou, qu'à l'inverse, des louanges publiques sont adressées à des collègues; ce que fit Félix Gaillard à Gustave de Closmadeuc ${ }^{31}$ sur cette problématique. Cette histoire de dunes pourrait faire sourire, si elle ne s'inscrivait avant l'heure dans les travaux très actuels sur l'évolution du littoral et l'ancienneté des dunes, ancienne et récente, développés par des géomorphologues, géographes et archéologues, que ce soit au sud du Finistère ${ }^{32}$, sur la presqu'île quiberonnaise ${ }^{33}$ ou en contexte insulaire (Hoëdic) ${ }^{34}$. Cette dune continentale et insulaire, aujourd'hui morte, témoigne de l'existence d'un cordon sableux plus méridional qui l'a alimentée ponctuellement lors de la baisse temporaire du niveau marin, au $\mathrm{II}^{\mathrm{e}}$ millénaire av. J.-C. Cet épisode est à ce jour bien référencé et les sites archéologiques recouverts par cet horizon dunaire récent ne contiennent pas de vestiges antérieurs au début de l'âge du Bronze. Les modélisations renforcent cet argumentaire et signalent un épisode dunaire qui ne peut, à l'inverse, être postérieur. Cette idée avait déjà été évoquée, à l'image des conclusions poétiques et pleines de perspicacité de A. Guilcher qui en 1948 rappelait par la même occasion qu'il " faut toujours être sur ses gardes lorsqu'il s'agit de dater une forme en Bretagne. On a toujours tendance à croire les reliefs plus récents qu'ils ne le sont en réalité 35 ". À moins de deux kilomètres au nord de Port-Blanc, les coffres à inhumations de l'île Thinic (Portivy à Saint-Pierre-Quiberon)

31. GAILLARD, Félix., "Rapport sur les fouilles du cimetière celtique de l'île de Thinic (15 août 1883) ", Bulletin de la Société polymathique du Morbihan, n 27, 1883, p. 231-240.

32. Giot, Pierre-Roland, "La dune ancienne de la baie d'Audierne ", Norois, n 179, 1998, p. $487-494$.

33. Roul, Thierry, Les dépôts littoraux quaternaires fossiles de la presqu'île de Quiberon, mémoire de maîtrise multigraphié, IGARUN - Université de Nantes, 1991, 1 vol., 171 p.

34. BlanchaRD, Audrey, "Occupations insulaires au Néolithique récent : Groah Denn 1 à Hoëdic (Morbihan) ", Annales de Bretagne et des Pays d'Ouest, n 119, 1, 2012, p. 7-30.

35. GullCHER, André, Le relief de la Bretagne méridionale de la baie de Douarnenez à la Vilaine, thèse de doctorat multigraphiée, La Roche-sur-Yon, 1948, 682 p., 113 fig., 8 cartes, 89 pl. (cf. p. 601). 
ont été fouillés par Félix Gaillard ${ }^{36}$ en août 1883, quelques mois après le complexe mégalithique qui nous intéresse ici, puis en 1916 par Zacharie Le Rouzic ${ }^{37}$. Une nouvelle fois, la découverte puis la fouille de Thinic tiennent au fait que ces anciennes architectures de pierres étaient clairement recouvertes par la dune récente. Le mobilier, abondant, est alors rapproché des productions lithiques et céramiques reconnues notamment dans les monuments mégalithiques néolithiques. Malgré ces données primordiales, tant stratigraphiques que matérielles, offertes par le fouilleur, parfois le site de Thinic apparaît encore dans certains textes - mais fort heureusement pas tous - comme étant possiblement daté entre l'âge du Bronze et la fin du premier âge du $\mathrm{Fer}^{38}$, et ce n'est pas un cas isolé. Histoires de marchands de sable...

Le deuxième point d'accroche chronologique est relatif au mobilier découvert à Port-Blanc, actuellement dispersé dans trois lieux de conservation $^{39}$ et qui a fait l'objet de plusieurs études (figure 7). Il est regrettable que l'étude ne puisse ici porter que sur le mobilier issu presque exclusivement de l'intérieur des architectures mégalithiques. Lors de fouilles modernes, une attention particulière aurait aussi été portée aux façades du cairn et à l'extérieur du complexe, ce qui n'a pas été le cas à la fin du XIX ${ }^{\mathrm{e}}$ siècle. Le lot le plus diversifié provient du niveau supérieur de la chambre du monument $\mathrm{A}$, où ont été retrouvés les deux derniers inhumés en connexion anatomique. En plus de l'épingle en os et du poinçon en bronze retrouvés chacun près d'un des corps, il faut ajouter la découverte d'un grain de collier, d'une défense de sanglier, de deux lames de haches polies en dolérite, d'un éclat de silex et d'un "vase apode caliciforme ", qui renvoie à une fréquentation campaniforme, certes ponctuelle, de cette seule tombe à couloir, à la fin du III /début du II ${ }^{\mathrm{e}}$ millénaire av. J.-C. Le fouilleur n'a pas vu de séparation nette (couche sédimentaire, pierres, etc.) entre ces deux inhumés et les premiers ossements du niveau supérieur néolithique : il y a donc bien deux niveaux sépulcraux néolithiques, dont le dernier est perturbé par la suite par les campaniformes. Cela explique aussi l'hétérogénéité du mobilier associé, avec des objets nouveaux (poinçon, vase, etc.) et anciens (lames de haches, pendeloque, etc.). Le reste du mobilier est associé aux niveaux néolithiques, notamment une pendeloque (galet perforé) et un grand vase découvert dans le monument B ou les deux vases trouvés près de nombreux crânes (niveaux supérieur et inférieur) dans le couloir

36. GAILlARD, Félix, "Rapport sur les fouilles du cimetière celtique de l'île de Thinic (15 août 1883) ", Bulletin de la Société polymathique du Morbihan, n 27, 1883, p. 231-240.

37. LE RouzIC, Zacharie, « Morphologie et chronologie des sépultures préhistoriques du Morbihan ", L’Anthropologie, 43, n³-4, 1933, p. 225-265; LE RouZIC, Zacharie, " Le mobilier des sépultures préhistoriques du Morbihan ", L’Anthropologie, 44, 1934, p. 485-524.

38. VILlard-Le Tiec, Anne, Lorho, Thierry, MagiterRi, Caroline, " Ploemeur, Kerham - LannPorz-Menec'h (Morbihan) : un monument funéraire du premier âge du Fer? ", Revue archéologique de l'Ouest, $\mathrm{n}^{\circ}$ 24, 2007, p. 31-53.

39. Le Musée d'Archéologie Nationale de Saint-Germain-en-Laye (Yvelines), le Musée d'Histoire et d'Archéologie de Vannes et le Musée de Préhistoire de Carnac (Morbihan). 
Figure 7 - Mobilier recueilli dans le niveau supérieur de la tombe à couloir A (d'après Gaillard, 1883)
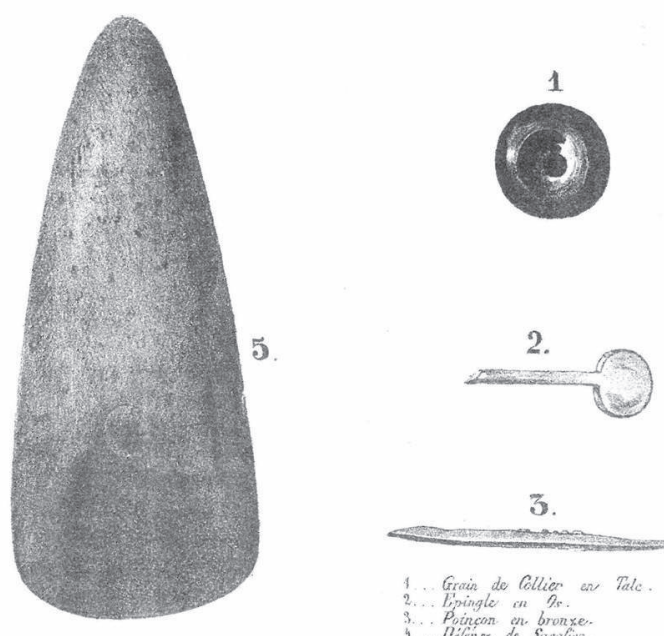

3.
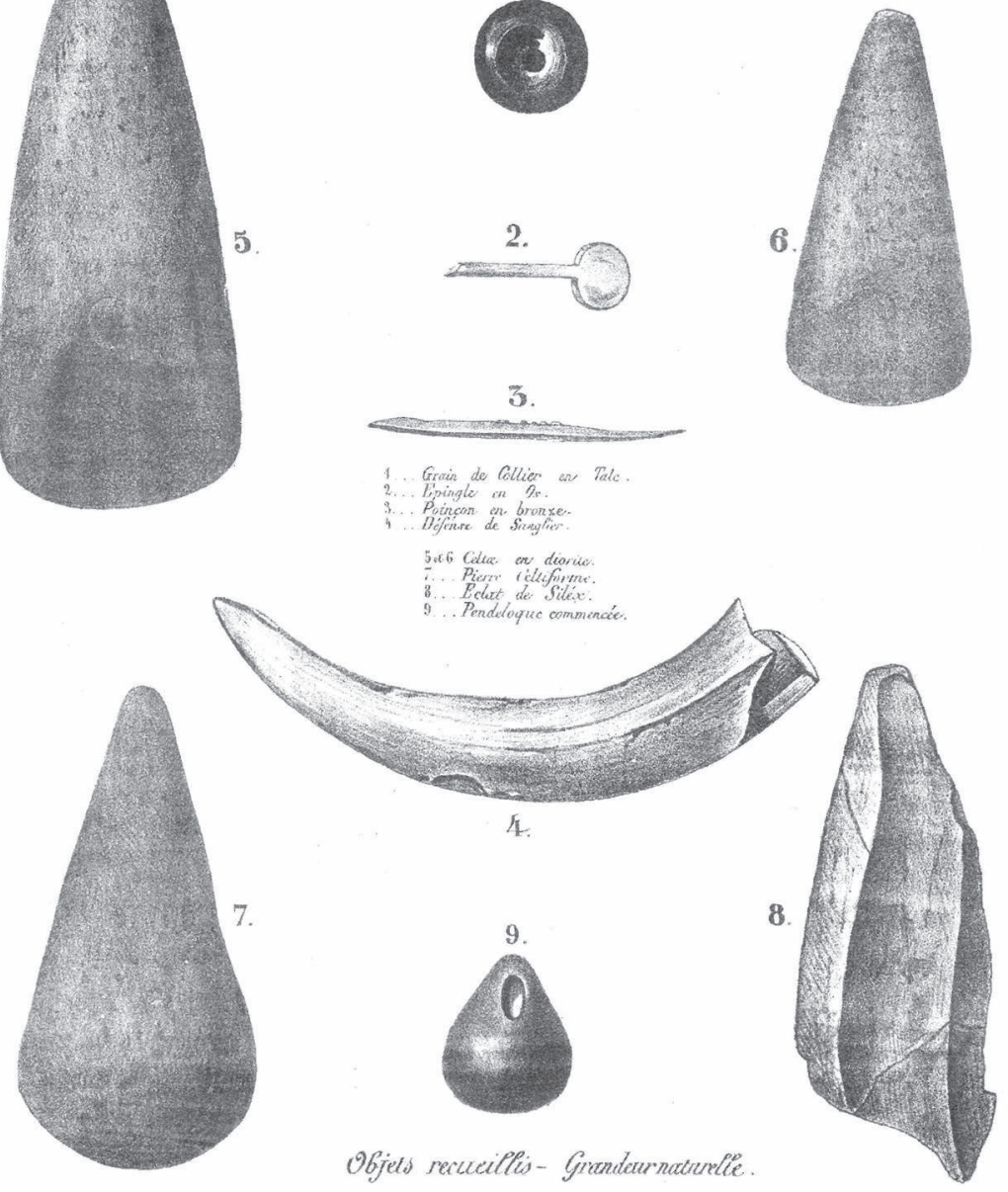

du A. Le mobilier de la tranchée exploratoire (C) accompagnant un grand nombre de restes osseux - dont un fragment de lame de hache, des percuteurs et au moins deux vases dont un orné - a été rapidement interprété par le fouilleur comme les vestiges d'une vidange du dolmen A, distant de $5 \mathrm{~m}$. Le premier récipient de petites dimensions à profil ové, au bord rentrant à lèvre amincie et surface lustrée, comporte un motif de triangles hachurés emboîtés en partie supérieur (figure 8). Un autre fragment provient d'un vase à fond plat dont la paroi est relativement fine $(4 \mathrm{~mm})$, à surface 
Figure 8 - Vase à profil ové orné de cannelures découvert près de l'aire dallée de la tranchée exploratoire $C$ (d'après Pollès, 1986, fig. 4; collection Musée de Vannes)

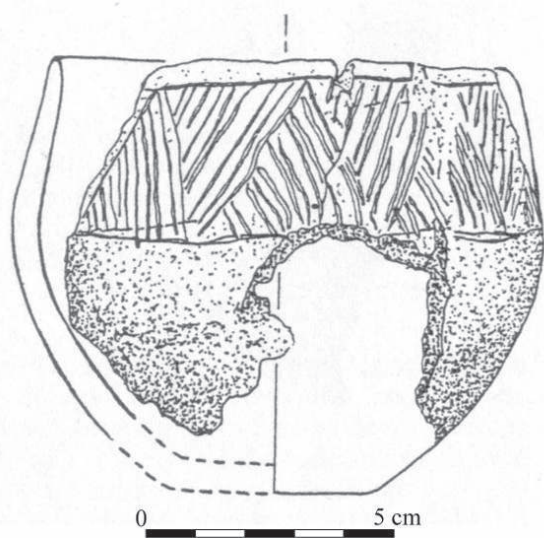

brune et irrégulière et brune. D'autres fragments de bords, droits à éversés, aux lèvres arrondies, amincies ou encore aplanies, ainsi qu'un fond plat, complètent ce lot céramique. L'assemblage lithique est dominé par des galets côtiers de silex et quelques fragments de quartz. Hormis les esquilles, les différents éléments constitutifs de la chaîne opératoire sont présents : blocs bruts, galets testés, demi-galets, nucléus, éclats, et une lame, débités par percussion directe dure, voire posée sur enclume. Les outils, essentiellement quelques pièces esquillées sur nucléus et éclats, sont peu nombreux. Le macro-outillage recueilli dans cette même tranchée $\mathrm{C}$ renvoie à diverses activités, certaines domestiques, travail du bois (éclat de lame de hache polie en roche indéterminée), débitage (enclume en quartz, percuteurs), d'autres non, extraction de matière minérale (coin en quartz, percuteurs). Il semblerait donc que ce secteur, à proximité du bloc couché, ne soit pas qu'un dépôt de restes humains - dont le statut du rassemblement ("vidange " selon les termes de Félix Gaillard) est d'ailleurs inconnu - mais qu'il fut dans un premier temps une aire d'activités vraisemblablement en lien avec la phase de construction mégalithique. Malgré l'absence d'évocation de l'état et des formes du substrat de la part du fouilleur, il est possible d'envisager qu'il y ait eu dans ce secteur, en association avec ces outils spécifiques, une zone d'extraction (carrière) et d'acquisition des matériaux nécessaires pour la construction de ces monuments. Ce cas ne serait d'ailleurs pas isolé puisque le proche site d'habitat littoral de Groh-Collé (Saint-Pierre-Quiberon) offre, en arrière de l'architecture talutée, une carrière d'extraction de moellons associée à un outillage composé de pics, coins et percuteurs ${ }^{40}$.

Pour synthétiser, les céramiques à fonds plats plaident en faveur de fréquentations postérieures au Néolithique moyen II. Le récipient orné issu de la tranchée exploratoire s'intègre parfaitement au contexte régional du début du Néolithique récent. Le profil ové du récipient et son motif décoratif renvoient indéniablement au style Conguel ${ }^{41}$. La forme a ainsi des correspondances avec les récipients rencontrés sur le site éponyme

40. GuYodo, Jean-Noël, L'habitat néolithique de Groh-Collé..., op. cit..

41. BlanchaRD, Audrey, Le Néolithique récent de l'Ouest de la France..., op. cit. 
de Conguel (niveau supérieur; Quiberon, Morbihan) ${ }^{42}$, dans le dolmen de Butten-er-Hah (Groix, Morbihan), dans le dolmen du Rocher (Le Bono, Morbihan $)^{43}$, ou encore sur l'alignement mégalithique de Groah Denn 1 (Hoëdic, Morbihan) ${ }^{44}$. Le motif cannelé, Conguel, de triangles hachurés imbriqués en partie haute du récipient est peu fréquent; seuls des récipients de Butten-er-Hah, de Kercado et quelques fragments de Groah Denn 1 en sont ornés. Un autre vase orné de Port Blanc est publié par Paul Du Chatellier ${ }^{45}$ en 1897 mais semble avoir disparu lorsque Renan Pollès reprend l'étude en 1986. Il se caractérisait par une forme basse, à fond rond et à large col orné de deux bandes superposées de triangles imbriqués portant des hachures alternées. Si la forme dénote quelque peu, elle n'en est pas moins proche de certains récipients Groh-Collé. Le motif décoratif proposé est lui bien connu sur le site de Kercado. L'assemblage lithique réellement issu des monuments se cantonne aux deux lames de haches polies en dolérite et à l'éclat de silex mentionnés par Félix Gaillard; pièces ubiquistes difficiles à raccorder à une sphère chrono-culturelle. Néanmoins, l'assemblage recueilli, alentour du monument, n'est pas sans rappeler le Néolithique récent régional. L'utilisation de galets côtiers de silex, ou encore de quartz, matières premières locales, est un point commun aux gisements Groh-Collé, tout comme l'usage répétée de la percussion posée sur enclume. Le façonnage de pièces esquillées sur nucléus est également un trait caractéristique de ces productions, qu'on retrouve en quantité sur les proches gisements de Groh-Collé (Saint-Pierre-Quiberon, Morbihan), d'Er Yoh (Houat, Morbihan) ${ }^{46}$, de Groah Denn (Hoëdic, Morbihan) ou encore de l'îlot de Guernic (Saint-Pierre-Quiberon, Morbihan) ${ }^{47}$.

La dernière source d'inspiration pour cerner la chronologie tient aux récentes datations par le radiocarbone réalisées par R. Schulting ${ }^{48}$ sur quelques restes humains provenant de contextes variés (tableau 1). Le crâne trépané (appelé Broca 265) provenant assurément du niveau inférieur du dolmen A est ainsi daté de 3930-3660 Cal BC, confirmant la première utilisation - et peut-être la construction - de la tombe à couloir la plus

42. L'Helgouac'H, Jean, "Le dolmen de Conguel en Quiberon (Morbihan) ", Bulletin de la Société préhistorique française, 59, 5-6, 1962, p. 371-381.

43. PollÈs, Renan, "Le style de Conguel : nouveaux éléments ", Bulletin de la Société préhistorique française, 83, 11-12, 1986, p. 452-469.

44. BlanchARD, Audrey, "Occupations insulaires au Néolithique récent... ", art. cit.

45. Du Chatellier, Paul, La poterie aux époques préhistoriques et gauloises en Armorique, Rennes et Paris, Plihon et Hervé, 1897, 60 p.

46. GuYODO, Jean-Noël, "Installations néolithiques et gauloises à Er Yoc'h (Houat, Morbihan) : état de la question ", Melvan, La Revue des deux îles, 4, 2007, p. 229-250.

47. GuYodo, Jean-Noël, "L'atelier de débitage de Guernic (Saint-Pierre-Quiberon, Morbihan) : résultats des campagnes 1998-1999 ", Bulletin de l'AMARAI, 13, 2000, p. 43-64.

48. Schulting, Rick, " Comme la mer qui se retire : les changements dans l'exploitation des ressources marines du Mésolithique au Néolithique en Bretagne ", dans : Grégor MARCHAND, Anne TRESSET (dir.), Unité et diversité des processus de néolithisation sur la façade atlantique de l'Europe ( $6^{e}-4^{e}$ millénaire av. J.-C.), Actes de la table ronde, Nantes, 2002, Société préhistorique française, mémoire 36, 2005, p. 163-171. 
récente à la fin du Néolithique moyen II ou au début du Néolithique récent. Le mobilier évoqué plus haut - bien que discret - suggère plutôt cette seule dernière ambiance. Les deux derniers fragments osseux datés n'ont pu être contextualisés précisément, ce qui est fort regrettable. Néanmoins, l'un d'eux (dit R82.31.1) offre une datation (3950-3710 Cal BC) sensiblement proche de la précédente tandis que le second os humain, daté de 2870-2590 $\mathrm{Cal} \mathrm{BC}$, renvoie à un fonctionnement plus récent de la structure.

\section{Tableau 1 - Datations par le radiocarbone, sites de Port-Blanc et Groh-Collé (Saint-Pierre-Quiberon)}

\begin{tabular}{|l|c|c|c|}
\hline \multicolumn{1}{|c|}{ Échantillon } & Date B.P. & $\begin{array}{c}\text { Date calibrée BC } \\
(2 \text { sigmas })\end{array}$ & Laboratoire \\
\hline Port-Blanc R82.31.1 & $5070+/-50$ & $3950-3710$ & OxA-10615 \\
\hline Port-Blanc Broca 265 & $5050+/-40$ & $3930-3660$ & OxA-10936 \\
\hline Port-Blanc R82.31.25 & $4200+/-45$ & $2870-2590$ & OxA-10699 \\
\hline $\begin{array}{l}\text { Groh-Collé, talus, } \\
\text { phase récente }\end{array}$ & $4230+/-35$ & $2911-2681$ & Lyon-8381 (GrA) \\
\hline $\begin{array}{l}\text { Groh-Collé talus, } \\
\text { phase ancienne }\end{array}$ & $4325+/-35$ & $3023-2889$ & Lyon-8380 (GrA) \\
\hline
\end{tabular}

\section{En définitive...}

Les études récentes et les données architecturales inédites offertes ici dévoilent un peu plus les comportements humains anciens, les choix techniques et/ou culturels et en définitive l'histoire sociale. L'originalité tient à l'exploitation et à la gestion de matériaux de construction prélevés sur l'estran. L'emploi intentionnel d'un imposant galet marin - faisant ici office de dalle de chevet dans la chambre - est également sensible dans la proche tombe à couloir de Roch en Aud (ou Roch'enn aud), toujours à Saint-Pierre-Quiberon, prise dans l'urbanisme et véritablement coincée entre plusieurs maisons, ce déjà lors des premières fouilles et explorations. Ni Gatien Chapelain-Duparc qui l'explora le premier en 1877 - mais qui ne put atteindre le fond de la chambre - ni Félix Gaillard, dont des écrits relatent la restauration du monument menée en août $1889^{49}$, ne l'indiquent. Pourtant, un cliché de Zacharie Le Rouzic, daté du début du xx ${ }^{e}$ siècle, assure que ce bloc est bien en position primaire puisque les dalles et piliers de l'architecture sont clairement en place. De même forme architecturale qu'à Port-Blanc, la tombe à couloir de Roch en Aud possède une vaste chambre mégalithique $\left(16 \mathrm{~m}^{2}\right)$, presque carrée $(3,90 \times 4 \mathrm{~m})$, couverte de dalles mégalithiques. Le dallage est posé sur les aspérités du rocher naturel. Le couloir semble très court $(1,10 \mathrm{~m})$ mais aurait pu, sans certitude

49. GAILlARD, Félix, "La restauration du dolmen de Roch'enn aud en Saint-Pierre ", Bulletin de la Société d'anthropologie de Paris, III 'érie, tome 12, 1889, p. 125-128. 
toutefois, être plus long d'après Félix Gaillard. Rien ne permet de dater clairement cette architecture du Néolithique moyen plus que du Néolithique récent puisque, comme de nombreux autres monuments régionaux ${ }^{50}$, l'intérieur a été fortement bouleversé par une fréquentation gallo-romaine, pour laquelle certains vestiges sont mentionnés (nombreux débris de poteries, tuile à rebord $)^{51}$. Ce système de mise en valeur de dalle de chevet de la tombe à couloir de Roch en Aud, par le choix d'un matériau différent de celui des autres orthostates, n'est pas particulièrement original à l'échelle régionale : la Table des Marchands à Locmariaquer (Morbihan) ${ }^{52}$ est sans doute l'un des plus célèbres parmi les nombreux exemples connus. Le fait qu'il s'agisse d'un imposant galet marin est plus original, mais cela renvoie au choix opéré sur d'autres sites quiberonnais, funéraires (Port-Blanc) et domestiques (Groh-Collé). Néanmoins, dans le cas présent, la distance site/ estran est la plus importante, de l'ordre de 1,5 kilomètre si l'on prend en considération le rivage le plus proche (côte occidentale ou nord-occidentale) offrant ce type de matériau.

À Port-Blanc, les tombes à couloir A et B, proches dans le temps en terme de construction, attestent un changement de stratégie d'approvisionnement en matériaux, extraits pour la plus ancienne (B), en partie prélevés de façon privilégiée sur l'estran (galets marins et piédestaux à forme d'érosion marine) pour la plus récente (A). Reste à savoir si ce phénomène relève d'une intention particulière... Une partie de la réponse pourrait venir des contextes domestiques, souvent évacués des travaux sur l'architecture. À Groh-Collé, un talus de pierres ceinture l'espace domestique de 0,5 hectare et ferme une pointe littorale de la Côte sauvage quiberonnaise. Entre les parements de pierres sèches qui en constituent les limites extérieures, le comblement central est en partie composé de nombreux galets marins mesurant parfois jusqu'à un mètre de longueur. Cette observation ne concerne que la phase ancienne de la construction puisque le talus est dans un second temps rehaussé et élargi, désormais avec des blocs uniquement extraits de carrières. Si l'on se réfère aux récentes datations par le radiocarbone effectuées sur ce site d'habitat, la phase récente est en partie contemporaine de la deuxième phase de Port-Blanc (tombe à couloir A et dépôt d'ossements $\mathrm{C}$ ) et la culture matérielle recueillie similaire.

50. ARs, Edith, "Les figurines en terre cuite du Morbihan ", Bulletin de la Société polymathique du Morbihan, 1997, p. 41-54; GALLIOU, Patrick, «Le passé recomposé. Les réutilisations de monuments du Néolithique et de l'âge du Bronze en Armorique à l'âge du Fer et à l'époque romaine ", Cahiers de l'Iroise, n 208, 2009, p. 97-132; Rutin, Yohan, Les réoccupations d'époque romaine dans les monuments mégalithiques du Grand-Ouest, mémoire de master 1 Archéologie et Histoire, Univ. Rennes 2, 2011, 1 vol.

51. GaILlaRD, Félix, "La restauration du dolmen de Roch'enn aud en Saint-Pierre ", Bulletin de la Société d'anthropologie de Paris, III éérie, tome 12, 1889, p. 125-128, cf. p. 127.

52. CASSEn, Serge, (dir.), Autour de la Table. Explorations archéologiques et discours savants sur des architectures néolithiques à Locmariaquer, Morbihan (Table des Marchands et Grand Menhir) : Synthèse d'un programme de fouilles (J. L'Helgouac'h et S. Cassen, 1986-1994) et d'une action collective de recherche (ACR) 2003-2006, Nantes, Université de Nantes, LARA, $918 \mathrm{p}$. 
Sachant que les deux sites sont distants de deux kilomètres et visibles l'un de l'autre, on pourrait voir ici un lien entre un habitat domestique ceinturé et un complexe sépulcral. Être presque assuré d'une telle relation est extrêmement rare. L'habitat ceinturé (1,8 hectare) contemporain du Lizo à Carnac offre un autre cas de proximité affirmé par la présence, en partie centrale du site, d'une tombe à couloir, certes bâtie au Néolithique moyen II mais dont l'utilisation comme espace sépulcral perdure au Néolithique récent, lors de l'occupation domestique. Ce lien géographique n'a toutefois pu être corrélé par des datations par le radiocarbone, inexistantes en l'état actuel.

Si l'on reprend le découpage chronologique et la terminologie récemment définis pour le Nord-Ouest de la France ${ }^{53}$, il est désormais possible d'ordonner les informations et d'évoquer des changements techniques et des stratégies d'approvisionnement multiples, comme autant de possibilités offertes aux groupes culturels, à l'échelle micro-régionale. L'exploitation du milieu minéral revêt trois formes particulières : extraction par prélèvement d'affleurements rocheux (substrat émergeant), extraction en profondeur dans le substrat (carrières) et prélèvement de galets marins sur l'estran (blocs naturellement détachés). Durant le Néolithique récent I (3800/3700-3400/3300 Cal BC), notamment durant sa phase ancienne, ces trois formes d'exploitation sont mises en œuvre pour la construction de la tombe à couloir de Roch en Aud et de la tombe à couloir A de Port-Blanc (niveau sépulcral inférieur daté de 3930-3660 Cal BC). Pour la plus ancienne (tombe à couloir B; niveau sépulcral daté de 3950-3710 Cal BC), seule concernée par l'alternance piliers dressés/parements de petites dalles ${ }^{54}$, aucun bloc prélevé sur l'estran n'est à signaler. Évidemment cette remarque est à pondérer en raison du faible nombre de monolithes encore en place. Toutefois, ce type d'architecture particulier (piliers/parements) offrirait sans doute peu de place à des blocs roulés. Comme pour la période précédente, les habitats domestiques font malheureusement cruellement défaut pour cette phase chronologique, et pas seulement dans ce secteur puisque l'Ouest de la France est encore loin d'offrir un quelconque référentiel. Le site littoral et insulaire de la Pointe de la Tranche (île d'Yeu), dont la fouille programmée a débuté en 2010 (dir. A. Blanchard), est un habitat domestique ceinturé par une architecture de pierre (type talus) conservée en élévation, avec sols anciens associés, daté par le radiocarbone (3400-3100 Cal BC) ${ }^{55}$ et attribué au Néolithique récent II (3400/3300-3 100/3000 Cal $\mathrm{BC})$. Les trois techniques architecturales y ont été reconnues, tout comme sur le proche site contemporain de Ker Daniaud (île d'Yeu), puisque les dalles dressées et les blocs formant les murets de pierre sèche ont été extraits de carrières et d'affleurements rocheux, ainsi que prélevés sur l'es-

53. Blanchard, Audrey, Le Néolithique récent de l'Ouest de la France..., op. cit.

54. L'Helgouac'H, Jean, Les sépultures mégalithiques en Armorique..., op. cit., cf. p. 50.

55. Blanchard, Audrey, L'éperon barré néolithique de la Pointe de la Tranche (Île d'Yeu, Vendée), rapport de fouille programmée, SRA de Pays de la Loire, Nantes, 2012, 62 p. 
tran (galets marins, dont certains régularisés, mais pas de piédestal). Les données concernant les monuments funéraires sont ici éludées, faute de contextes clairement datés. Enfin, il en va de même durant le Néolithique récent III (3 100/3000-2900/2800 Cal BC) : la diversité des sources d'approvisionnement perdure, avec toutefois un bémol concernant l'exploitation, moins fréquente, des blocs marins, présents à Groh-Collé phase ancienne (3023-2889 Cal BC) mais absents des architectures en pierre des habitats domestiques de Pen Men et de Groh-Collé phase récente (2911-2680 Cal $\mathrm{BC}$ ). Si, comme le suggère Félix Gaillard, la plateforme (annexe C) de PortBlanc servant d'aire sépulcrale externalisée est bien contemporaine du niveau d'inhumations supérieur de Port-Blanc A (2870-2590 Cal BC), il en va de même puisqu'il n'y aurait ici que des blocs extraits formant le dallage découvert en 1883.

Sur cette base, bien que le Néolithique récent soit désormais partagé en trois phases avec autant de groupes culturels, définis notamment par la culture matérielle, qui se succèdent, les stratégies d'approvisionnement, les traditions ainsi que le bagage technique des bâtisseurs sont presque identiques. De plus, sur cette aire géographique restreinte, littorale et insulaire, l'usage de la pierre ne permet pas de démontrer qu'il puisse y avoir de stratégie d'approvisionnement en matière première minérale, de gestion différenciée des matériaux et de technique de construction réservées aux seules architectures funéraires ou domestiques. Il est ainsi difficile d'appréhender la dynamique interne (étapes, mécanismes) de ces cultures par la caractérisation et la hiérarchisation des gisements à travers l'exploitation de l'environnement minéral. Trois possibilités sont offertes, extraction par prélèvement d'affleurements rocheux, extraction en profondeur (carrières) et prélèvement sur l'estran, sans que l'on soit en mesure d'identifier clairement la genèse de la dernière puisque les sites, antérieurs, du Néolithique moyen II régional n'offrent pas de réelle information quant à l'exploitation des blocs d'estran, glanés (galets imposants) ou prélevés (piédestaux, blocs à érosion marine partielle). L'apport de la lecture technologique, comme analyse des méthodes et techniques de construction, a permis d'affiner le phasage du complexe mégalithique de Port-Blanc et sa relation avec d'autres sites à l'échelle micro-régionale. Son originalité est l'extraction et l'intégration de grands blocs à érosion marine ce qui est bien moins fréquent, à l'échelle régionale dans la construction funéraire et/ou domestique, que l'utilisation d'imposants galets marins. Plus que la forme des piédestaux qui aurait été privilégiée, c'est peut-être l'intérêt architectonique pour la construction de la chambre et le maintien de sa couverture qu'il faut ici envisager, d'autant que ces deux blocs sont disposés sur les piliers de la chambre et du couloir, côté nord, et qu'ils auraient pu faciliter la construction de la voûte en encorbellement de la tombe à couloir A, dont ils serviraient d'assise basale. Ce choix n'est donc pas culturel mais sans doute personnel, en tant que réponse à un problème technique, de la part de bâtisseurs. 
Il est donc illusoire, car impossible, de vouloir profiter des seules provenances et formes des matériaux pour définir un phasage ancien/récent du début $\mathrm{du}_{\mathrm{IV}}^{\mathrm{e}}$ au début du $\mathrm{III}^{\mathrm{e}}$ millénaire av. J.-C. pour une datation, et par là une typologie, des sites tant funéraires que domestiques; sachant de surcroît que le statut des sites n'entre pas en ligne de compte. Les changements fréquents mais infimes de modalités d'approvisionnement plus que de traditions et de bagage technique pourraient tout autant relever d'effets de mode plus que de réels choix culturels. Le fait le plus évident est que les ressources minérales d'estran sont toujours engagées, mais moins utilisées alors que toujours abondantes et accessibles au Néolithique récent III (3100/3000-2900/2800 Cal BC). Les modalités de l'exploitation du milieu minéral (acquisition et économie de la matière première) offrent certes un indice complémentaire sur la tradition technique architecturale, l'histoire de la construction et l'histoire sociale mais ne peuvent véritablement entrer ici en ligne de compte pour la définition du schème technique des groupes culturels du Néolithique récent de l'Ouest de la France, puisque peu perceptibles.

\section{RÉSUMÉ}

Au nord-ouest de la presqu'île quiberonnaise, l'ensemble mégalithique de Port-Blanc (ou Porz Guen) à Saint-Pierre-Quiberon (Morbihan) occupe un rebord de falaise. À la fin du xIxe siècle, Félix Gaillard le fouille méticuleusement, suite à la visite nocturne de quelques chercheurs de trésor. Au cours de cette opération archéologique, de nombreux os humains, parfois en connexion, ont été découverts; fait relativement rare dans l'Ouest de la France. À l'occasion de la récente restauration de ce monument, des observations complémentaires et de nouveaux relevés ont pu être effectués. Cette étude architecturale (matériaux utilisés, techniques employées, etc.) offre des informations inédites. Celles-ci méritent ici d'être croisées avec les diverses données accumulées au cours des 130 années qui se sont écoulées depuis le début de la première intervention de terrain, un certain dimanche 18 février 1883...

\section{ABSTRACT}

To the northwest of the Quiberon peninsula, the megalithic group of Port-Blanc (or Porz Guen) at Saint-Pierre-Quiberon (Morbihan) occupies a cliff edge. At the end of nineteenth century, further to the night-visit of some treasure hunters, it was fastidiously excavated by Félix Gaillard. During this archaeological dig, some human bones, sometimes connected, were discovered; a relatively rare occurrence in Western France. With the recent restoration of this monument, additional research has been undertaken and new samples taken. This architectural study (raw material, techniques of construction, etc.) offers new information that deserves to be cross-analysed with the data accumulated over the 130 years since the beginning of the first intervention, on Sunday 18th February 1883... 